\title{
Coordinating Parallel Mobile Ambients to Solve SAT Problem in Polynomial Number of Steps
}

\author{
Bogdan Aman and Gabriel Ciobanu \\ Romanian Academy, Institute of Computer Science, Iaşi, Romania \\ "A.I.Cuza" University, Blvd. Carol I no.11, 700506 Iaşi, Romania \\ gabriel@info.uaic.ro, bogdan.aman@gmail.com
}

\begin{abstract}
In this paper we present a version of mobile ambients, called parMA, having a weak form of replication and a parallel semantics. We investigate how parMA can solve intractable problems in a polynomial number of computational steps. We use parMA to give a semiuniform solution to a well-known strong NP-complete problem, namely to the Boolean satisfiability problem (SAT).
\end{abstract}

\section{Introduction}

Ambient calculus is a formalism for describing distributed and mobile computing [11. In contrast with the $\pi$-calculus [19] where mobility is expressed by communication, the ambient calculus uses an explicit notion of movement given by moving actions (in and out) together with an "opening" action and (local) communication. An ambient is a named location, and it represents the unit of movement. The authors of [11] introduce the mobile ambients as "a paradigm of mobility where computational ambients are hierarchically structured, where agents are confined to ambients and where ambients move under the control of agents". Their initial goal was "to make mobile computation scale-up to widely distributed, intermittently connected and well administered computational environments". The resulting ambient model is elegant and powerful, well suited for expressing issues of mobile computations as working environment, allowing access to information or resources [2]6. Many variants have been proposed; among them, we mention mobile safe ambients [17, push and pull ambient calculus [16] and boxed ambients [7. Despite the fact that the initial motivation of mobile ambients assumes a high degree of parallelism in their evolution, the usual semantics of the proposed variants is the interleaving semantics.

Some results show that mobile ambients have the computational power of Turing machines by encoding into ambient calculus (or fragments of it) some formalisms known to be Turing complete: asynchronous $\pi$-calculus [1112], counter machines [9] and Turing machines [11:18]. A link between mobile ambients and $\pi$-calculus is established in [13, where it is proven that pure mobile ambients can be embedded into a fragment of the $\pi$-calculus, namely in the localized sum-free synchronous monadic $\pi$-calculus with matching and mismatching. Other authors relate ambients to security issues and to system biology. Some simulators were 
also developed [14 in which the evolution of mobile ambients can be observed easily (see http://www-sop.inria.fr/mimosa/ambicobjs/).

Many formal machine models (e.g., Turing machines) have an infinite number of memory locations. Mobile ambients are computing devices of finite size having a finite description with a fixed amount of initial resources (ambients and processes), that can evolve to a possibly infinite family of mobile ambients obtained by replication in order to solve a (decision) problem. A decision problem $X$ is a pair $\left(I_{X}, \theta_{X}\right)$ such that $I_{X}$ is a language over a finite alphabet (whose elements are called instances) and $\theta_{X}$ is a total boolean function (that is, a predicate) over $I_{X}$. Its solvability is defined through the recognition of the language associated with it. Let $M$ be a Turing machine with the working alphabet $\Gamma, L$ a language over $\Gamma$, and the result of any halting computation is yes or no. If $M$ is a deterministic device, it recognizes or decides $L$ whenever, for any string $u$ over $\Gamma$, if $u \in L$, then $M$ accepts $u$ (the result on input $u$ is yes), or $M$ rejects $u$ (the result on input $u$ is no). If $M$ is a non-deterministic device, it recognizes or decides $L$ whenever, if for any string $u$ over $\Gamma, u \in L$, and only if there exists a computation of $M$ with input $u$ such that the answer is yes.

According to [15], the NP-complete problems are divided into weak (e.g., Knapsack) and strong (e.g., SAT) depending on the size of the input. We show how a parallel version of mobile ambients with a weak form of replication can solve NP-complete problems in a polynomial number of steps. We provide a semiuniform solution to the best known strong NP-complete problem (SAT) in a polynomial number of steps [22. To give such a solution, we treat mobile ambients as "deciding devices" that respect the following conditions: (1) all computations halt, (2) two special names yes and no are used, and (3) in a halting configuration a channel ans is ready to output one of the names yes and no; the computation is accepting if yes is present in the halting configuration, and rejecting if no is present in the halting configuration on channel ans. Mobile ambients respect these conditions if we impose some constraints:

- We use a true concurrent semantics allowing processes to run in parallel. The key rule is

$$
P \rightarrow P^{\prime} \text { and } Q \rightarrow Q^{\prime} \text { implies } P\left|Q \rightarrow P^{\prime}\right| Q^{\prime} .
$$

This parallel semantics is natural if we recall that in 11] a process is described as "running even when the surrounding ambient is moving", aspect which the interleaving semantics does not reflect properly. Other reasons to consider a parallel semantics are presented in [14] where the authors present a distributed implementation of mobile ambients.

- A restricted form of replication is used by considering a weaker duplication operator which only doubles a process; this means that a reduction rule $! P \rightarrow P \mid P$ is used instead of the congruence rule $! P \equiv P \mid ! P$ or instead of the reduction rule $! P \rightarrow P \mid \quad ! P$. This duplication rule is also used by R. Milner in [20]. It helps in controlling all the computations to halt (and so fulfilling condition 1 . of deciding devices).

- We use a special symbol (S) that helps in delimiting the computational steps. (S) is a purely technical device that is used in the subsequent 
formalization of the structural operational semantics of parMA; intuitively, (S) $P$ specifies a process $P$ which is temporarily stalled and so cannot execute any action.

- We use two kinds of action rules: $\rightarrow$ and $\stackrel{\phi}{\Rightarrow}$. The former is an execution of a set of actions, and the latter is used to remove all occurrences of (S) (using a tree parsing algorithm) in order to start a new round of parallel actions.

The differences between parallel and interleaving semantics is underlined also in [5]: "The parallel construct is interpreted in terms of interleaving, as usual in many (timed) process algebras .... Alternatively one could adopt maximal parallelism, which means that at each moment every enabled process of the system is activated". In defining a parallel semantics, we follow the solution used also in [8] where such a semantic is defined in brane calculi [10], a process algebra related to BioAmbients [23].

The paper is organised as follow. Section 2 defines the syntax and semantics of parallel mobile ambients (parMA). In Section 3 we give some notions of complexity, and show how to solve SAT problem in a polynomial number of steps. Section 4 illustrates how mobile ambients compute effectively by considering a SAT instance with three clauses and three variables as an example. Conclusion and references end the paper.

\section{Parallel Mobile Ambients}

In this section we present a variant of mobile ambients having a parallel semantics. Initially, mobility in ambient calculus involved the authorization to enter or exit certain domains in order to access information; the access to information is controlled at many levels: local computer, local area network, regional area network, wide-area intranet and internet. We consider a framework given by mobile ambients hierarchically structured inside a well-defined environment, where ambients move under the control of agents running inside them.

\subsection{Syntax}

Table 1 describes the syntax of parMA.

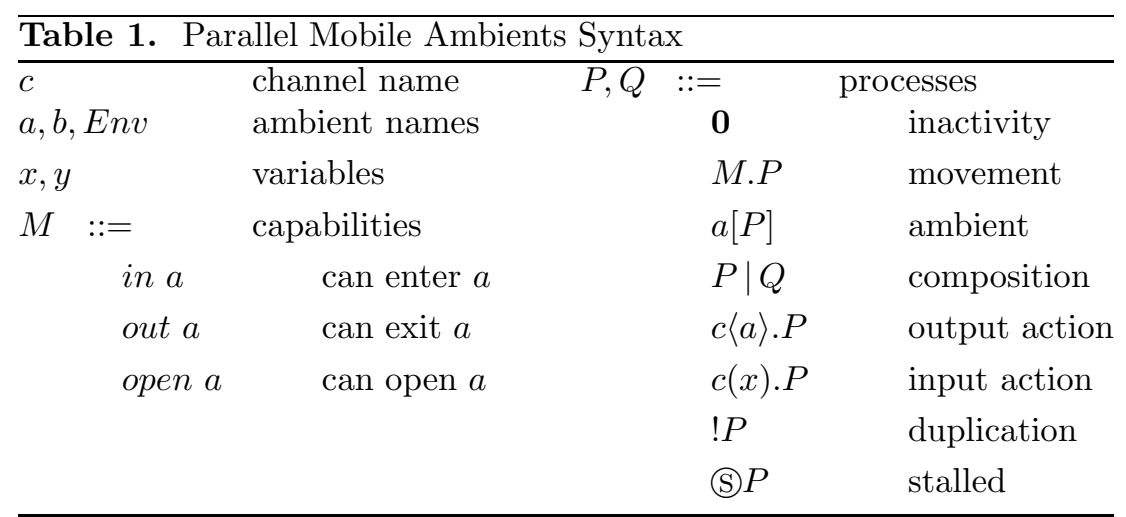


The name Env represents the environment in which the mobile ambients reside, and can appear only once, at the top of the hierarchical structure. Process 0 is an inactive process (it does nothing). A movement M.P is provided by the capability $M$, followed by the execution of process $P$. An ambient $a[P]$ represents a bounded place labelled by $a$ in which a process $P$ is executed. $P \mid Q$ is a parallel composition of processes $P$ and $Q$. An output action $c\langle a\rangle . P$ releases a name $a$ on channel $c$, and then behaves as process $P$. An input action $c(x) . P$ captures a name from channel $c$, and binds it to a variable $x$ within the scope of process $P$. A weak form of replication, namely the duplication of a process $P$ (producing two parallel copies of process $P$ ) is denoted by $! P$. The process (S) $P$ is used to state that process $P$ is temporarily "stalled".

\subsection{Operational Semantics}

The first component of the operational semantics of parMA is the structural congruence $\equiv$. It is the smallest congruence such that the equalities from Table 2 hold. Its role is to rearrange a process in order to apply the action rules given in Table 3. The axioms from Table 2 describe the commutativity and associativity of the parallel composition.

\begin{tabular}{ll}
\hline Table 2. & Structural Congruence \\
\hline$P \equiv P$ & $P|Q \equiv Q| P$ \\
$(P \mid Q)|R \equiv P|(Q \mid R)$ & $P \mid \mathbf{0} \equiv P$ \\
$! \mathbf{0} \equiv \mathbf{0}$ & \\
\hline
\end{tabular}

The set $f n(P)$ of free names of a process $P$ is defined as:

$$
f n(P)= \begin{cases}\emptyset & \text { if } P=\mathbf{0} \\ f n(R) \cup\{a\} & \text { if } P=\text { in a.R or } P=\text { out a.R } \\ & \text { or } P=\text { open a.R or } P=a[R] \\ f n(R) \cup\{a, c\} & \text { if } P=c\langle a\rangle \cdot R \\ f n(R) \cup\{c\} & \text { if } P=c(x) . R \\ f n(R) \cup f n(Q) & \text { if } P=R \mid Q \\ f n(R) & \text { if } P=! R\end{cases}
$$

Table 3 introduces two kinds of action rules: $P \rightarrow P^{\prime}$ and $P \stackrel{\phi}{\Rightarrow} P^{\prime}$. The former is an execution of a set of actions, and the latter is used to remove all occurrences of (S) in order to start a new round of parallel actions. The first five rules of Table 3 are one-step reductions for in, out, open, communication and duplication. In rule (Com), by $P^{\prime}\{a / x\}$ we denote the substitution by $a$ of each free occurrence of variable $x$ in process $P^{\prime}$. The next three rules propagate reductions across ambient nesting and parallel composition. In rule (Par2), by $R \nrightarrow \rightarrow$ is denoted a process $R$ that cannot evolve. Rule (Struct) allows the use of structural congruence during reduction. When no rule can be applied in Env, rule (Step) is used to delete all occurrences of (S) in order to start a new round of transitions. It can be noticed that in rules (Par2) and (Step) we use negative 
premises: an activity is performed based on the absence of actions. This is due to the fact that sequencing the evolution can only be defined using negative premises, as done for sequencing processes [421.

\begin{tabular}{ll}
\hline Table 3. Reduction Rules \\
\hline (In) & $\frac{a \neq E n v \quad b \neq E n v}{a[\text { in } b . P \mid Q] \mid b[R] \rightarrow b[a[\mathrm{~S} P \mid Q] \mid R]}$ \\
(Out) & $\frac{a \neq E n v \quad b \neq E n v}{a[b[\text { out } a . P \mid Q] \mid R] \rightarrow b[\mathrm{~S} P \mid Q] \mid a[R]}$ \\
(Open) & $\frac{a \neq E n v}{\text { open } a . P \mid a[Q] \rightarrow(\mathrm{S} P \mid Q}$ \\
(Com) & $c\langle a\rangle \cdot P\left|c(x) \cdot P^{\prime} \rightarrow \mathrm{S} P\right| \mathrm{S} P^{\prime}\{a / x\}$ \\
(Dupl) & $! P \rightarrow \mathrm{S} P \mid \mathrm{S} P$ \\
(Amb) & $\frac{P \rightarrow Q}{a[P] \rightarrow a[Q]}$ \\
(Par1) & $\frac{P \rightarrow P^{\prime} Q \rightarrow Q}{P\left|Q \rightarrow P^{\prime}\right| Q^{\prime}}$ \\
(Par2) & $\frac{P \rightarrow Q}{P|R \rightarrow Q| R}$ \\
(Struct) & $\frac{P^{\prime} \equiv P, P \rightarrow Q, \quad Q \equiv Q}{P^{\prime} \rightarrow Q^{\prime}}$ \\
(Step) & $\frac{E n v[P] \not \rightarrow}{P \stackrel{\phi}{\Rightarrow} \phi(P)}$ \\
\hline
\end{tabular}

The rules of Table 3 define execution of processes. A complete computational step in mobile ambients is captured by a derivation of the form

$$
\operatorname{Env}[P] \rightarrow \stackrel{\phi}{\Rightarrow} \operatorname{Env}\left[P^{\prime}\right] \text {. }
$$

This means that a derivation is a compressed representation of a sequence of individual actions followed by a reinitialization step (removing of all (S symbols).

\subsection{Example}

To illustrate the basic components of parMA, we use an example in which several students wish to move from the campus to the university and back, having the possibility to use either a tram or a bus. The scenario involves eight ambients and four processes.

$$
\begin{aligned}
& \operatorname{Env}\left[\text { campus } \left[\operatorname{student}\left[P_{1} \mid P_{4}\right] \mid \operatorname{student}\left[P_{1} \mid P_{4}\right]\right.\right. \\
& \left.\left.\left|\operatorname{tram}\left[P_{2} \mid P_{4}\right]\right| \text { bus }\left[P_{3} \mid P_{4}\right]\right] \mid \text { univ }\left[\operatorname{student}\left[P_{1} \mid P_{4}\right]\right]\right]
\end{aligned}
$$

The role of the ambients is suggested by their names. The processes are:

- $P_{1}=Q_{1} \mid Q_{2}$

$Q_{1}=$ in tram.c $(x) . c(x) . c(x) . o u t$ tram. $Q_{1}$

$Q_{2}=$ in bus.c $(x) . c(x) . c(x) . c(x)$.out bus. $Q_{2}$ 
- $P_{2}=c(x)$.out campus.c(x).in univ. $P_{2}^{\prime}$ $P_{2}^{\prime}=c(x) . o u t$ univ.c $(x)$.in campus. $P_{2}$

- $P_{3}=c(x)$.out campus.c $(x) . c(x)$.in univ. $P_{3}^{\prime}$ $P_{3}^{\prime}=c(x)$. out univ.c $(x) . c(x)$. in campus. $P_{3}$

- $P_{4}=c\langle a\rangle . P_{4}$

The communication on channel $c$, that takes place inside ambients, is used to model the fact that the tram, bus and students perform the following actions:

- the first $c(x)$ from $P_{2}, P_{2}^{\prime}, P_{3}$ and $P_{3}^{\prime}$ represents the fact that the bus and tram, once inside the campus or univ, are willing to wait for students that intend to travel between campus and univ.

- the others $c(x)$ from $P_{2}, P_{2}^{\prime}, P_{3}$ and $P_{3}^{\prime}$ are used to model the fact that the movement of the tram and bus between campus and univ takes a number of steps (equal with the number of input actions on channel $c$ ).

- all $c(x)$ from $Q_{1}$ and $Q_{2}$ are used to prevent the students from getting out of the tram or bus before reaching the desired location.

It can be noticed that both students from campus can enter either the bus or the tram. Suppose both choose the tram. Then the mobile ambient

Env $\left[\right.$ campus $\left[\right.$ student $\left[P_{1} \mid P_{4}\right] \mid$ student $\left[P_{1} \mid P_{4}\right]$

evolves to $\left.\left|\operatorname{tram}\left[P_{2} \mid P_{4}\right]\right| \operatorname{bus}\left[P_{3} \mid P_{4}\right]\right] \mid$ univ $\left.\left[\operatorname{student}\left[P_{1} \mid P_{4}\right]\right]\right]$

\section{Env $\left[\right.$ campus $\left[\right.$ tram $\left[\right.$ Sout campus.c $(x)$.in univ. $P_{2}^{\prime} \mid$ S $P_{4}$ \\ | student $\left[\mathrm{S} c(x) . c(x) . c(x)\right.$. out tram. $\left.Q_{1}\left|Q_{2}\right| \mathrm{S} P_{4}\right]$ | student $\left[\mathrm{S} c(x) \cdot c(x) \cdot c(x)\right.$. out tram. $\left.\left.Q_{1}\left|Q_{2}\right| \mathrm{S} P_{4}\right]\right]$}

| bus [Sout campus.c $(x) . c(x)$. in univ. $P_{3}^{\prime} \mid$ S $\left.\left.P_{4}\right]\right] \mid$ univ $\left[\right.$ student $\left.\left.\left[P_{1}\right]\right]\right]$

The tram and the bus are still inside campus since they communicated on channel $c$ in order to permit the willing students to get inside them. At this moment it can be noticed that only rule (Step) can be applied in order to eliminate the symbols (S), obtaining the mobile membrane

$$
\begin{gathered}
\text { Env }\left[\text { campus } \left[\operatorname { t r a m } \left[\text { out campus.c }(x) . \text { in } \text { univ. } P_{2}^{\prime} \mid P_{4}\right.\right.\right. \\
\mid \text { student }\left[c(x) . c(x) . c(x) . \text { out tram. } Q_{1}\left|Q_{2}\right| P_{4}\right] \\
\left.\mid \text { student }\left[c(x) . c(x) . c(x) . \text { out tram. } Q_{1}\left|Q_{2}\right| P_{4}\right]\right] \\
\left.\left.\mid \text { bus }\left[\text { out campus.c }(x) . c(x) . \text { in univ. } P_{3}^{\prime} \mid P_{4}\right]\right] \mid \text { univ }\left[\text { student }\left[P_{1}\right]\right]\right]
\end{gathered}
$$

After three steps, the tram is inside univ where it waits for the students to exit/enter it, while the bus is still between the two campus and univ, being ready to enter univ in the next step. Thus the next mobile ambient is obtained

$$
\begin{gathered}
\text { Env }\left[\text { campus } [ ] | \text { bus } [ \text { in univ. } P _ { 3 } ^ { \prime } | P _ { 4 } ] | \text { univ } \left[\operatorname { t r a m } \left[P_{2}^{\prime} \mid P_{4}\right.\right.\right. \\
\mid \text { student }\left[\text { out tram. } Q_{1}\left|Q_{2}\right| P_{4}\right] \\
\left.\left.\left.\mid \text { student }\left[\text { out tram. } Q_{1}\left|Q_{2}\right| P_{4}\right]\right] \mid \text { student }\left[P_{1}\right]\right]\right]
\end{gathered}
$$

In the next step the two students from the tram get out inside univ, while the student that was waiting enters the tram in order to reach campus. The obtained mobile ambients is

$$
\begin{gathered}
\text { Env }\left[\text { campus } [ ] | \text { univ } \left[\text { bus }\left[P_{3}^{\prime} \mid P_{4}\right] \mid \operatorname{tram}\left[\text { out univ.c }(x) \text {.in campus. } P_{2} \mid P_{4}\right.\right.\right. \\
\left.\mid \text { student }\left[c(x) . c(x) . c(x) . \text { out tram. } Q_{1}\left|Q_{2}\right| P_{4}\right]\right] \\
\left.\left.\left|\operatorname{student}\left[P_{1}\right]\right| \text { student }\left[P_{1}\right]\right]\right]
\end{gathered}
$$


The mobile ambient continues to change, but we stop here since we have illustrated the expressive power of the proposed formalism.

\section{Solving NP-Complete Problems in Polynomial Steps}

As stated in the introduction, we use mobile ambients as deciding devices, in which all computation starting from the initial ambient agree on the result. A family MA, a collection of ambients, solves a decision problem if for each instance of the problem there is a member of the family able to decide on the instance. In order to define the notions of (semi)uniformity, we denote:

- for a suitable alphabet $O$, each instance of the decision problem is encoded as a string $v$ over $O$;

- $V=\left\{v_{1}, \ldots\right\}$ - the language of encoded instances of the given problem;

- $\mathbf{M A}(v)$ - the member of MA which solves the instance $v$;

- $\mathbf{M} \mathbf{A}_{n}$ - the member of MA which solves all instances of length $n$.

Definition 1. The family MA

(i) decides $V$ if for any string $v \in O^{*}$, the mobile ambient $\mathbf{M A}(v)$ (or $\mathbf{M} \mathbf{A}_{n}$ for all instances $v,|v|=n$ ) generates an yes answer whenever $v \in V$ and a no answer otherwise;

(ii) is sound with respect to $V$ when, for any string $v \in O^{*}$, if there exists an accepting computation of $\mathbf{M A}(v)\left(\mathbf{M A}_{n}\right)$, then $v \in V$;

(iii) is complete with respect to $V$ when, for any string $v \in O^{*}$, if $v \in V$, then every computation of $\mathbf{M A}(v)$ ( $\left.\mathbf{M A}_{n}\right)$ is accepting.

Inspired by the uniformity conditions applied to families of Boolean circuits [6], we imposed similar ones on families of processes. By imposing certain resource restrictions (on number of steps and space) to the function that constructs each member of the family MA, it can be ensured that the set of problems decided by the family does not increase. The function is called an

- uniformity condition if an instance size is mapped to a mobile ambient that decides all instances of that length;

- semiuniformity condition if a single instance is mapped to a mobile ambient that decides that instance.

Definition 2. If we consider a set of problem instances $V=\left\{v_{1}, v_{2}, \ldots\right\}$, two classes of functions $E, F$ and a total function $t: \mathbb{N} \rightarrow \mathbb{N}$, such that:

1. there exist a F-uniform family of mobile ambients $\mathbf{M A}=\left\{\mathbf{M A}_{1}, \ldots\right\}$; this means that there exist a function $f \in F, f:\{1\}^{*} \rightarrow$ MA such that $f\left(1^{n}\right)=\mathbf{M A}_{n}$, namely all instances $v_{k}$ of length $n$ are solved by $\mathbf{M} \mathbf{A}_{n}$, where $\mathbf{M A}_{n}$ can be constructed by a function $f \in F$; 
2. there exists an encoding function $e \in E$ such that $e(v)$ is the input process of $\mathbf{M A}_{n}$, for $|v|=n$;

3. MA is t-efficient: $\mathbf{M} \mathbf{A}_{n}$ halts in $t(n)$ steps (e.g., $\mathbf{M A}$ is polynomial efficient if $t(n)$ is polynomial in $n$ for all $n$ );

4. MA is sound and complete with respect to $V$,

then we say that the class of problems $V$ is solved by an $(E, F)$-uniform family of mobile ambients $\mathbf{M A}$, and denote the family by $(E, F)$-MA $(t)$. The set of languages decided by a uniform family of mobile ambients in a polynomial number of steps is defined as $(E, F)$-PMA $=\bigcup_{k \in \mathbb{N}}(E, F)-\mathbf{M A}\left(n^{k}\right)$.

Semiuniformity is a generalization of uniformity, namely

Definition 3. If we consider a set of problem instances $V=\left\{v_{1}, v_{2}, \ldots\right\}$, $a$ class of functions $H$ and a total function $t: \mathbb{N} \rightarrow \mathbb{N}$, such that:

1. there exist a H-semiuniform family of mobile ambients $\mathbf{M A}=\left\{\mathbf{M A}_{v_{1}}\right.$, $\left.\mathbf{M A}_{v_{2}}, \ldots\right\}$; namely, there exist a function $h \in H, h: V \rightarrow \mathbf{M A}$ such that $h\left(v_{i}\right)=\mathbf{M A}_{v_{i}}$

2. MA is t-efficient: $\mathbf{M} \mathbf{A}_{n}$ halts in $t\left(\left|v_{n}\right|\right)$ steps;

3. MA is sound and complete with respect to $V$,

then we say that the class of problems $V$ is solved by an $(H)$-semiuniform family of mobile ambients $\mathbf{M A}$, and denote the family by $(H)-\mathbf{M A}(t)$. The set of languages decided by a semiuniform family of mobile ambients in a polynomial number of steps is defined as $(H)$-PMA $=\bigcup_{k \in \mathbb{N}}(H)-\mathbf{M A}\left(n^{k}\right)$.

\subsection{Boolean Satisfiability Problem}

The SAT problem checks the satisfiability of a propositional logic formula in conjunctive normal form (CNF). Let $\left\{x_{1}, x_{2}, \ldots, x_{n}\right\}$ be a set of Boolean variables. A formula in $\mathrm{CNF}$ is of the form

$$
\varphi=C_{1} \wedge C_{2} \wedge \cdots \wedge C_{m}
$$

where each $C_{i}, 1 \leq i \leq m$ is a disjunction of the form

$$
C_{i}=y_{1} \vee y_{2} \vee \cdots \vee y_{r}(r \leq n),
$$

where each $y_{j}$ is either a variable $x_{k}$ or its negation $\neg x_{k}$. In this section we show how, starting from a formula $\varphi$, to construct a process $P$ that provides a semiuniform solution to the SAT problem by using mobile ambients with parallel semantics and duplication (for an instance of SAT we construct a mobile ambient which decides it). We start with the process

$$
P=P_{1} \mid Q_{1}
$$

in which $P_{1}$ is used to provide the answer to the problem when placed in parallel with $Q_{1}$, a process that generates all possible assignments over the set $\left\{x_{1}, x_{2}, \ldots, x_{n}\right\}$ of Boolean variables. In what follows we describe how each of these two processes are constructed starting from the $\varphi$ formula. 
- process $Q_{1}$ is defined recursively using the processes $Q_{i}(1 \leq i<n)$ and $Q_{n}$. For each variable $x_{i}$ from the set $\left\{x_{1}, x_{2}, \ldots, x_{n}\right\}$ of Boolean variables, we construct a process $Q_{i}$ defined as follows:

where:

$$
\begin{gathered}
Q_{i}=x_{i}\left\langle t_{i}\right\rangle \cdot x\langle z\rangle \cdot x\langle z\rangle\left|x_{i}\left\langle f_{i}\right\rangle \cdot x\langle z\rangle . x\langle z\rangle\right| \\
\mid ! x_{i}\left(y_{i}\right) \cdot\left(x(y) . x(y) . \text { open } k_{i} \mid k_{i}\left[Q_{i+1}\right]\right), \text { for } 1 \leq i<n \\
Q_{n}=x_{n}\left\langle t_{n}\right\rangle\left|x_{n}\left\langle f_{n}\right\rangle\right| ! x_{n}\left(y_{n}\right) . A\left[x_{1}\left\langle y_{1}\right\rangle|\ldots| x_{n}\left\langle y_{n}\right\rangle\right. \\
\left.\left|y_{1}\langle a\rangle\right| \ldots\left|y_{1}\langle a\rangle\right| \ldots\left|y_{n}\langle a\rangle\right| \ldots\left|y_{n}\langle a\rangle\right| Q\right] .
\end{gathered}
$$

- process $Q$ contains terms of the form $t_{k}(b)$.in $C_{j}$ (if $x_{k}$ appears in $C_{j}$ ) or $f_{k}(b)$.in $C_{j}$ (if $\neg x_{k}$ appears in $C_{j}$ ). For example if we consider a $3 \mathrm{CNF}$ satisfiability problem with $\varphi=C_{1} \wedge C_{2} \wedge C_{3}$ and $X=\left\{x_{1}, x_{2}, x_{3}\right\}$, $C_{1}=x_{1} \vee \neg x_{3}, C_{2}=\neg x_{1} \vee \neg x_{2}$ and $C_{3}=x_{2}$ we have

$Q=t_{1}(b)$.in $C_{1} \mid f_{3}(b)$.in $C_{1} \mid f_{1}(b)$.in $C_{2} \mid f_{2}(b)$.in $C_{2} \mid t_{2}(b)$.in $C_{3}$

- each ambient $A$ will contain a different assignment over the set $\left\{x_{1}, x_{2}\right.$, $\left.\ldots, x_{n}\right\}$ of Boolean variables; after $Q_{n}$ is executed, there will be $2^{n}$ ambients $A$, obtained by using the duplication operator ! that proceeds the processes that generate an $A$ ambient.

- $y_{j}\langle a\rangle|\ldots| y_{j}\langle a\rangle$ stands for $m$ parallel processes $y_{j}\langle a\rangle$, one for each disjunction, and, after all $y_{j}$ are instantiated, are used to communicate with the processes from $Q$.

- $x\langle z\rangle \cdot x\langle z\rangle$ are used to introduce a delay that prevents that an ambient $k_{i}$ containing a $Q_{i+1}$ is not opened to soon and cause unwanted evolutions.

- process $P_{1}$ has the form:

where:

$$
\begin{gathered}
P_{1}=C_{1}\left[\ldots \left[C_{m}[J[x(y) \ldots x(y) \mid x\langle z\rangle \ldots x\langle z\rangle . K[\text { out } J]]\right.\right. \\
\mid L[\text { in A.ans }\langle\text { yes }\rangle \mid \text { in K.ans }\langle\text { no }\rangle]]]]
\end{gathered}
$$

- to each disjunction $C_{i}, 1 \leq i \leq m$ we associate an ambient $C_{i}$;

- the ambients $C_{i}, 1 \leq i \leq m$ are placed one inside the other, forming an ambient structure of depth $m$. The order in which these ambients are placed is not important (thus the construction is not unique), but for simplicity we consider the ambient

$$
C_{1}\left[\ldots\left[C_{m}[\ldots]\right] \ldots\right]
$$

- the previous ambient is used to check if there exists an assignments over the set $\left\{x_{1}, x_{2}, \ldots, x_{n}\right\}$ of Boolean variables that respects all these disjunctions. If such an assignment exists, this means that an ambient $A$ containing this assignment, will eventually reach inside ambient $C_{m}$ (an ambient $A$ enters an ambient $C_{i}$ if the assignment placed inside $A$ respect the disjunction $C_{i}$ )

- $x(y) \ldots x(y)$ stands for a $2 n+m+1$ sequence of capabilities $x(y)$, and together with $x\langle z\rangle \ldots x\langle z\rangle$ that stands for a $2 n+m+1$ sequence of capabilities $x\langle z\rangle$, introduces a delay equal with the number of steps needed by an ambient $A$ to get inside ambient $C_{m}$, before the ambient $K$ exits ambient $J$. It can be noticed that if an ambient $A$ gets near the ambient $L$ (inside ambient $C_{m}$ ), this ambient enters the ambient $A$, generates the yes answer and prevents $K$ to generate the negative answer (the ambient $K$ cannot interact with the ambient $L$ inside the ambient $A$ ). 
In what follows we explain how these two processes $\left(P_{1}\right.$ and $\left.Q_{1}\right)$ once constructed from the $\varphi$ formula, can generate an answer to the problem.

Starting from $P_{1}$ the evolution in the first $2 n+1$ steps is given by the rule $P_{i} \rightarrow \stackrel{\phi}{\Rightarrow} P_{i+1}$, where $P_{i+1}$ is obtained from $P_{i}$ by performing a communication on channel $x$. In parallel, starting from the process $Q_{1}$, are generated $2^{n}$ ambients $A$ that contain all possible assignments over the variables $\left\{x_{1}, \ldots, x_{n}\right\}$, namely each assignment is contained inside an ambient $A$.

Next we describe in detail the evolution of $Q_{1}$. We have two cases.

Case 1: For $1 \leq i \leq n-1$, the evolution of each

$$
\begin{gathered}
Q_{i}=x_{i}\left\langle t_{i}\right\rangle \cdot x\langle z\rangle \cdot x\langle z\rangle \mid x_{i}\left\langle f_{i}\right\rangle \cdot x\langle z\rangle . x\langle z\rangle \\
\mid ! x_{i}\left(y_{i}\right) \cdot\left(x(y) . x(y) . \text { open } k_{i} \mid k_{i}\left[Q_{i+1}\right]\right)
\end{gathered}
$$

from the $2^{i-1}$ processes $Q_{i}$ running in parallel, starts with a duplication, because any other reduction is not possible. The process that duplicates is in fact the process containing the ambient labelled by $k_{i}$ in which the process $Q_{i+1}$ is placed. This is done because the variables $y_{1}, \ldots, y_{i-1}$, $i \geq 2$ are already instantiated in the process $Q_{i+1}$, and we want to create two new copies: one in which $y_{i}$ is replaced by $t_{i}$, and one in which $y_{i}$ is replaced by $f_{i}$, keeping also the already instantiated variables. We obtain the process:

$$
\begin{gathered}
Q_{i}^{1}=\mathbf{x}_{\mathbf{i}}\left\langle\mathbf{t}_{\mathbf{i}}\right\rangle \cdot x\langle z\rangle \cdot x\langle z\rangle \mid \mathbf{x}_{\mathbf{i}}\left\langle\mathbf{f}_{\mathbf{i}}\right\rangle \cdot x\langle z\rangle \cdot x\langle z\rangle \\
\mid \mathbf{x}_{\mathbf{i}}\left(\mathbf{y}_{\mathbf{i}}\right) \cdot\left(x(y) \cdot x(y) \text {.open } k_{i} \mid k_{i}\left[Q_{i+1}\right]\right) \\
\mid \mathbf{x}_{\mathbf{i}}\left(\mathbf{y}_{\mathbf{i}}\right) \cdot\left(x(y) \cdot x(y) \text {.open } k_{i} \mid k_{i}\left[Q_{i+1}\right]\right)
\end{gathered}
$$

At this moment $Q_{i}^{1}$ has two input actions on channel $x_{i}$, and two output actions on channel $x_{i}$ that are ready to communicate the values of $t_{i}$ and $f_{i}$. This means that two (Comm) rules are applied in parallel, leading to:

$$
\begin{gathered}
Q_{i}^{2}=\mathbf{x}\langle\mathbf{z}\rangle \cdot x\langle z\rangle \mid \mathbf{x}\langle\mathbf{z}\rangle \cdot x\langle z\rangle \\
\mid \mathbf{x}(\mathbf{y}) \cdot x(y) \text {.open } k_{i} \mid k_{i}\left[Q_{i+1}\left\{t_{i} / y_{i}\right\}\right] \\
\mid \mathbf{x}(\mathbf{y}) \cdot x(y) \text {.open } k_{i} \mid k_{i}\left[Q_{i+1}\left\{f_{i} / y_{i}\right\}\right]
\end{gathered}
$$

After the communications on channels $x_{i}$ are performed, the communication of $t_{i+1}$ and $f_{i+1}$ inside $Q_{i+1}$ on channels $x_{i+1}$ takes place in two steps. This motivates a delay in opening the ambients $k_{i}$, such that the communication from all $k_{i}$ running in parallel does not get mixed up on channels $x_{i+1}$, leading to some unwanted assignments. The obtained process is:

$$
\begin{gathered}
Q_{i}^{3}=\mathbf{x}\langle\mathbf{z}\rangle|\mathbf{x}\langle\mathbf{z}\rangle| \mathbf{x}(\mathbf{y}) \text {.open } k_{i} \mid k_{i}\left[Q_{i+1}^{1}\left\{t_{i} / y_{i}\right\}\right] \\
\mid \mathbf{x}(\mathbf{y}) \text {.open } k_{i} \mid k_{i}\left[Q_{i+1}^{1}\left\{f_{i} / y_{i}\right\}\right]
\end{gathered}
$$

The channels $x_{i+1}$ are ready to communicate inside the processes $Q_{i+1}$, and so the capabilities open $k_{i}$ are released in the next step.

$$
Q_{i}^{4}=\text { open } \mathbf{k}_{\mathbf{i}}\left|\mathbf{k}_{\mathbf{i}}\left[Q_{i+1}^{2}\left\{t_{i} / y_{i}\right\}\right]\right| \text { open } \mathbf{k}_{\mathbf{i}} \mid \mathbf{k}_{\mathbf{i}}\left[Q_{i+1}^{2}\left\{f_{i} / y_{i}\right\}\right]
$$

Once the communication inside ambients $k_{i}$ on channels $x_{i+1}$ has finished, these ambients are opened, thus obtaining 


$$
Q_{i}^{5}=Q_{i+1}^{3}\left\{t_{i} / y_{i}\right\} \mid Q_{i+1}^{3}\left\{f_{i} / y_{i}\right\}
$$

Since the process $Q_{i}^{5}$ does not contain any capabilities or replication operators, except the ones from $Q_{i+1}^{3}$, it means that each $Q_{i}$ evolves for 5 steps, from which 3 steps are in parallel with the ones from $Q_{i+1}$.

Case 2: for $i=n$ the evolution of each

$$
\begin{gathered}
Q_{n}=x_{n}\left\langle t_{n}\right\rangle \mid x_{n}\left\langle f_{n}\right\rangle \\
\mid ! x_{n}\left(y_{n}\right) . A\left[x_{1}\left\langle y_{1}\right\rangle\left|\ldots x_{n}\left\langle y_{n}\right\rangle\right| y_{1}\langle a\rangle\left|\ldots y_{1}\langle a\rangle\right| \ldots y_{n}\langle a\rangle|\ldots| y_{n}\langle a\rangle \mid Q\right] \\
\text { from the } 2^{n-1} \text { processes } Q_{n} \text { running in parallel, starts with a duplication } \\
\text { rule, obtaining } \\
Q_{n}^{1}=\mathbf{x}_{\mathbf{n}}\left\langle\mathbf{t}_{\mathbf{n}}\right\rangle \mid \mathbf{x}_{\mathbf{n}}\left\langle\mathbf{f}_{\mathbf{n}}\right\rangle \\
\mid \mathbf{x}_{\mathbf{n}}\left(\mathbf{y}_{\mathbf{n}}\right) . A\left[x_{1}\left\langle y_{1}\right\rangle\left|\ldots x_{n}\left\langle y_{n}\right\rangle\right| y_{1}\langle a\rangle\left|\ldots y_{1}\langle a\rangle\right| \ldots y_{n}\langle a\rangle|\ldots| y_{n}\langle a\rangle \mid Q\right] \\
\mid \mathbf{x}_{\mathbf{n}}\left(\mathbf{y}_{\mathbf{n}}\right) . A\left[x_{1}\left\langle y_{1}\right\rangle\left|\ldots x_{n}\left\langle y_{n}\right\rangle\right| y_{1}\langle a\rangle\left|\ldots y_{1}\langle a\rangle\right| \ldots y_{n}\langle a\rangle|\ldots| y_{n}\langle a\rangle \mid Q\right] \\
\text { and after communications of } t_{n} \text { or } f_{n} \text { on channels } x_{n}: \\
Q_{n}^{2}=A\left[x_{1}\left\langle y_{1}\right\rangle\left|\ldots x_{n}\left\langle y_{n}\right\rangle\right| y_{1}\langle a\rangle\left|\ldots y_{1}\langle a\rangle\right| \ldots y_{n}\langle a\rangle|\ldots| y_{n}\langle a\rangle \mid Q\right]\left\{t_{n} / y_{n}\right\} \\
\mid A\left[x_{1}\left\langle y_{1}\right\rangle\left|\ldots x_{n}\left\langle y_{n}\right\rangle\right| y_{1}\langle a\rangle\left|\ldots y_{1}\langle a\rangle\right| \ldots y_{n}\langle a\rangle|\ldots| y_{n}\langle a\rangle \mid Q\right]\left\{f_{n} / y_{n}\right\}
\end{gathered}
$$

Since there are only ambients labelled by $A$ and no open capabilities, the process $Q_{n}^{2}$ cannot evolve any more. However, in order to cope with the fact that $Q_{i}^{5}=Q_{i+1}^{3}\left\{t_{i} / y_{i}\right\} \mid Q_{i+1}^{3}\left\{f_{i} / y_{i}\right\}$, for $1 \leq i \leq n-1$, we consider $Q_{n}^{2}$ to be equal with $Q_{n}^{3}$.

Starting from $P_{1} \mid Q_{1}$, after $2 n+1$ steps we obtain $P_{2 n+2} \mid Q^{\prime \prime}$, where:

$$
\begin{aligned}
& Q^{\prime \prime}=A\left[x_{1}\left\langle t_{1}\right\rangle|\ldots| x_{n}\left\langle t_{n}\right\rangle\left|t_{1}\langle a\rangle\right| \ldots\left|t_{1}\langle a\rangle\right| \ldots\left|t_{n}\langle a\rangle\right| \ldots\left|t_{n}\langle a\rangle\right| Q\right] \\
& \quad|\ldots| A\left[x_{1}\left\langle f_{1}\right\rangle|\ldots| x_{n}\left\langle f_{n}\right\rangle\left|f_{1}\langle a\rangle\right| \ldots\left|f_{1}\langle a\rangle\right| \ldots\left|f_{n}\langle a\rangle\right| \ldots\left|f_{n}\langle a\rangle\right| Q\right]
\end{aligned}
$$

To illustrate how such a process $Q^{\prime \prime}$ looks, we give a small example in which $n=m=2$, we have $2^{2}=4$ ambients generated by $Q_{1}$, and process $Q^{\prime \prime}$ is:

$$
\begin{aligned}
& Q^{\prime \prime}=A\left[x_{1}\left\langle t_{1}\right\rangle\left|x_{2}\left\langle t_{2}\right\rangle\right| t_{1}\langle a\rangle\left|t_{1}\langle a\rangle\right| t_{2}\langle a\rangle\left|t_{2}\langle a\rangle\right| Q\right] \\
& \mid A\left[x_{1}\left\langle t_{1}\right\rangle\left|x_{2}\left\langle f_{2}\right\rangle\right| t_{1}\langle a\rangle\left|t_{1}\langle a\rangle\right| f_{2}\langle a\rangle\left|f_{2}\langle a\rangle\right| Q\right] \\
& \mid A\left[x_{1}\left\langle f_{1}\right\rangle\left|x_{2}\left\langle t_{2}\right\rangle\right| f_{1}\langle a\rangle\left|f_{1}\langle a\rangle\right| t_{2}\langle a\rangle\left|t_{2}\langle a\rangle\right| Q\right] \\
& \mid A\left[x_{1}\left\langle f_{1}\right\rangle\left|x_{2}\left\langle f_{2}\right\rangle\right| f_{1}\langle a\rangle\left|f_{1}\langle a\rangle\right| f_{2}\langle a\rangle\left|f_{2}\langle a\rangle\right| Q\right]
\end{aligned}
$$

As it can be noticed, the four ambients $A$ contain all the Boolean assignments over the variables $\left\{x_{1}, x_{2}\right\}$, namely $\left\{t_{1}, t_{2}\right\},\left\{t_{1}, f_{2}\right\},\left\{f_{1}, t_{2}\right\},\left\{f_{1}, f_{2}\right\}$, and each possible assignment $t_{i}$ and $f_{i}$ is kept as an output value on channel $x_{i}$.

After obtaining all possible assignments, we need to check which one satisfies all the clauses $C_{j}$. To do this, we use the processes $Q$ that contain either terms of the form $t_{k}(b)$.in $C_{j}$ meaning that $x_{k}$ appears in $C_{j}$, or of the form $f_{k}(b) . i n C_{j}$ meaning that $\neg x_{k}$ appears in $C_{j}$. To be able to use the capability in $C_{j}$, there should be a $t_{k}\langle a\rangle$, respectively a $f_{k}\langle a\rangle$ inside the ambient $A$, both resulting from the instantiation of $y_{k}$. All ambients that satisfy the clause $C_{j}$ enter in parallel the ambient $C_{j}$. If there exist at least one ambient $A$ that contains in $C_{1}|\ldots|$ in $C_{m}$, it means that this ambient can go inside ambient $C_{m}$, and contains a solution to the SAT problem; in this case the ambient $L$ enters the ambient $A$ placed inside membrane $C_{m}$, releasing the yes answer on channel ans (1 step). Otherwise, ambient $K$ exits ambient $J$, and so ambient $L$ enters $K$; thus the no answer is send on channel ans (2 steps). 
We have a deterministic evolution of the mobile ambients, and so no interference (redex overlapping) exists in our solution of SAT problem. This motivates the use of mobile ambients rather than safe mobile ambients [17.

\subsection{Analysis}

If $n$ is the number of variables $\left(x_{1}, \ldots, x_{n}\right)$, and $m$ is the number of clauses $\left(C_{1}, \ldots, C_{m}\right)$, then the number of ambients, capabilities and duplication operators in the initial process is given by the sum of:

- 3 ambients and $4 n+2 m+7$ capabilities in process $P_{1}$;

- 10 capabilities, 1 ambient and 1 replication operator in each $Q_{i}, 1 \leq i<n$;

- $n+3+m n$ capabilities, 1 ambient and 1 replication operator in $Q_{n}$;

- maximum $4 m$ capabilities in $Q$.

Thus, the total size of the initial process is $\mathcal{O}(m n)$. The maximum number of computational steps performed in an execution is equal with $2 n+m+3$, a number determined by:

- $2 n+1$ steps to generate all the possible Boolean assignments over a set of variable $\left\{x_{1}, \ldots, x_{n}\right\}$

- $m$ steps required by a solution to move inside ambient $C_{m}$;

- either 1 step to generate a yes answer on channel ans, or 2 steps to generate a no answer on channel ans.

It is straightforward to show that:

- the construction of $P_{1} \mid Q_{1}$ is semiuniform;

- sound and complete: $P_{1} \mid Q_{1}$ says yes iff the given SAT instance is satisfiable;

- function $H$ required for the above construction is in $\mathbf{P}$.

Proposition 1. Using parMA, NP-complete problems can be solved in a polynomial number of steps.

\section{An Example of How Mobile Ambients Solve 3CNF-SAT}

To illustrate how mobility can "compute" and solve hard problems, we consider a $3 \mathrm{CNF}$ satisfiability problem with $\varphi=C_{1} \wedge C_{2} \wedge C_{3}$ and $X=\left\{x_{1}, x_{2}, x_{3}\right\}$, $C_{1}=x_{1} \vee \neg x_{3}, C_{2}=\neg x_{1} \vee \neg x_{2}$ and $C_{3}=x_{2}$. In this case $n=3$ and $m=3$. We start with the mobile ambient:

where

$$
P=P_{1} \mid Q_{1}
$$

$$
\begin{gathered}
P_{1}=C_{1}\left[\ldots \left[C_{3}[J[x(y) \ldots x(y) \mid x\langle z\rangle \ldots x\langle z\rangle . K[\text { out } J]]\right.\right. \\
\mid L[\text { in A.ans }\langle\text { yes }\rangle \mid \text { in K.ans }\langle\text { no }\rangle]]]]
\end{gathered}
$$

with $Q_{i}(1 \leq i<n)$ and $Q_{n}$ defined as follows

$$
Q_{i}=x_{i}\left\langle t_{i}\right\rangle \cdot x\langle z\rangle . x\langle z\rangle\left|x_{i}\left\langle f_{i}\right\rangle \cdot x\langle z\rangle \cdot x\langle z\rangle\right|
$$




$$
\begin{gathered}
\mid ! x_{i}\left(y_{i}\right) \cdot\left(x(y) . x(y) . \text { open } k_{i} \mid k_{i}\left[Q_{i+1}\right]\right), \text { for } 1 \leq i<3 \\
Q_{3}=x_{3}\left\langle t_{3}\right\rangle\left|x_{3}\left\langle f_{3}\right\rangle\right| ! x_{3}\left(y_{3}\right) . A\left[x_{1}\left\langle y_{1}\right\rangle\left|x_{2}\left\langle y_{2}\right\rangle\right| x_{3}\left\langle y_{3}\right\rangle\right. \\
\left|y_{1}\langle a\rangle\right| y_{1}\langle a\rangle\left|y_{1}\langle a\rangle\right| y_{2}\langle a\rangle\left|y_{2}\langle a\rangle\right| y_{2}\langle a\rangle\left|y_{3}\langle a\rangle\right| y_{3}\langle a\rangle \mid y_{3}\langle a\rangle \\
\left.\mid t_{1}(b) . \text { in } C_{1}\left|f_{3}(b) . i n C_{1}\right| f_{1}(b) . \text { in } C_{2} \mid f_{2}(b) . \text { in } C_{2} \mid t_{2}(b) . \text { in } C_{3}\right]
\end{gathered}
$$

The evolution of this term (by applying duplication and communication rules) leads in the first $2 n+1=2 * 3+1=7$ steps to the generation of all the possible truth assignments over a set of variables $\left\{x_{1}, x_{2}, x_{3}\right)$. Since we have described in the previous section how $Q_{1}$ evolves to $Q_{1}^{5}$, here we just enumerate the first five obtained ambients. In what follows we bold the capabilities and ambients involved actively in an evolution step.

$$
P_{1}\left|Q_{1} \rightarrow \stackrel{\phi}{\Rightarrow} \ldots \rightarrow \stackrel{\phi}{\Rightarrow} P_{6}\right| Q_{1}^{5}
$$

We replace $Q_{1}^{5}$ with $Q_{2}^{3}\left\{t_{1} / y_{1}\right\} \mid Q_{2}^{3}\left\{f_{1} / y_{1}\right\}$ obtaining

$$
P_{6}\left|Q_{2}^{3}\left\{t_{1} / y_{1}\right\}\right| Q_{2}^{3}\left\{f_{1} / y_{1}\right\}
$$

and then we substitute $Q_{2}^{3}$ processes for obtaining

$$
P_{6}|\mathbf{x}\langle\mathbf{z}\rangle| \mathbf{x}\langle\mathbf{z}\rangle|\mathbf{x}\langle\mathbf{z}\rangle| \mathbf{x}\langle\mathbf{z}\rangle
$$

$\mid \mathbf{x}(\mathbf{y})$.open $k_{2}\left|k_{2}\left[Q_{3}^{1}\left\{t_{1} / y_{1}, t_{2} / y_{2}\right\}\right]\right| \mathbf{x}(\mathbf{y})$.open $\left.k_{2} \mid k_{2}\left[Q_{3}^{1}\left\{t_{1} / y_{1}, f_{2} / y_{2}\right\}\right]\right)$

$\mid \mathbf{x}(\mathbf{y})$.open $k_{2}\left|k_{2}\left[Q_{3}^{1}\left\{f_{1} / y_{1}, t_{2} / y_{2}\right\}\right]\right| \mathbf{x}(\mathbf{y})$.open $\left.k_{2} \mid k_{2}\left[Q_{3}^{1}\left\{f_{1} / y_{1}, f_{2} / y_{2}\right\}\right]\right)$

In the next step the communication on all channels $x$ takes place in parallel, leading to all the possible assignments placed inside ambients $A$.

$\rightarrow \stackrel{\phi}{\Rightarrow} P_{7} \mid$ open $k_{2}\left|k_{2}\left[Q_{3}^{2}\left\{t_{1} / y_{1}, t_{2} / y_{2}\right\}\right]\right|$ open $k_{2} \mid k_{2}\left[Q_{3}^{2}\left\{t_{1} / y_{1}, f_{2} / y_{2}\right\}\right]$

open $k_{2}\left|k_{2}\left[Q_{3}^{2}\left\{f_{1} / y_{1}, t_{2} / y_{2}\right\}\right]\right|$ open $k_{2} \mid k_{2}\left[Q_{3}^{2}\left\{f_{1} / y_{1}, f_{2} / y_{2}\right\}\right]$

We replace $Q_{3}^{2}$ processes in order to see how the assignments look.

$$
\begin{array}{r}
P_{7} \mid \text { open } \mathbf{k}_{\mathbf{2}} \mid \mathbf{k}_{\mathbf{2}}\left[A\left\{t_{1} / y_{1}, t_{2} / y_{2}, t_{3} / y_{3}\right\} \mid A\left\{t_{1} / y_{1}, t_{2} / y_{2}, f_{3} / y_{3}\right\}\right] \\
\mid \text { open } \mathbf{k}_{\mathbf{2}} \mid \mathbf{k}_{2}\left[A\left\{t_{1} / y_{1}, f_{2} / y_{2}, t_{3} / y_{3}\right\} \mid A\left\{t_{1} / y_{1}, f_{2} / y_{2}, f_{3} / y_{3}\right\}\right] \\
\mid \text { open } \mathbf{k}_{\mathbf{2}} \mid \mathbf{k}_{2}\left[A\left\{f_{1} / y_{1}, t_{2} / y_{2}, t_{3} / y_{3}\right\} \mid A\left\{f_{1} / y_{1}, t_{2} / y_{2}, f_{3} / y_{3}\right\}\right] \\
\mid \text { open } \mathbf{k}_{\mathbf{2}} \mid \mathbf{k}_{\mathbf{2}}\left[A\left\{f_{1} / y_{1}, f_{2} / y_{2}, t_{3} / y_{3}\right\} \mid A\left\{f_{1} / y_{1}, f_{2} / y_{2}, f_{3} / y_{3}\right\}\right]
\end{array}
$$

where $A=A\left[x_{1}\left\langle y_{1}\right\rangle\left|x_{2}\left\langle y_{2}\right\rangle\right| x_{3}\left\langle y_{3}\right\rangle\right.$

$\left|y_{1}\langle a\rangle\right| y_{1}\langle a\rangle\left|y_{1}\langle a\rangle\right| y_{2}\langle a\rangle\left|y_{2}\langle a\rangle\right| y_{2}\langle a\rangle\left|y_{3}\langle a\rangle\right| y_{3}\langle a\rangle \mid y_{3}\langle a\rangle$

$\mid t_{1}(b)$.in $C_{1} \mid f_{3}(b)$.in $C_{1} \mid f_{1}(b)$.in $C_{2} \mid f_{2}(b)$.in $C_{2} \mid t_{2}(b)$.in $\left.C_{3}\right]$.

From this point forward, the performed steps are:

- Since all the possible assignments are generated, we open in parallel all ambients $k_{2}$ such that all ambients $A$ become siblings with the ambient $C_{1}$, ready to start the checking stage. Also all possible communications inside ambients $A$ are performed, thus launching the in $C_{j}$ capabilities corresponding to the clauses $C_{j}$.

- In the next $m=3$ steps, the solutions of $\varphi$ should go inside ambient $C_{3}$. First, the solutions of $C_{1}$ go inside ambient $C_{1}$ in parallel.

- Next, from the solutions of $C_{1}$ are selected the solutions of $C_{2}$, namely the solutions are all moved inside ambient $C_{2}$ in parallel.

- Finally, the solutions of $C_{3}$ are selected among the solutions of $C_{1}$ and $C_{2}$, namely the solutions which move in parallel inside ambient $C_{3}$. 
- Since we have an ambient $A$ inside ambient $C_{3}$, an yes answer is released on channel ans in the next step. In parallel, ambient $K$ comes out of ambient $J$, but since ambient $L$ is not present, the no answer cannot be sent on channel ans.

- Alternatively, if after $2 n+m+1$ steps there is no ambient $A$ inside ambient $C_{3}$, then ambient $K$ exits ambient $J$, and so allowing the ambient $L$ to enter ambient $K$ and to release a no answer on channel ans.

\section{Conclusion}

There are a large number of process calculi used to model complex systems in which interactions and mobility are essential (e.g., [3]). Following this research line, we have previously extended mobile ambients with timers [2] and types [1] in order to study their ability of modelling complex systems in distributed networks. In this paper we use mobile ambients with a parallel semantic (parMA) in order to study their complexity aspects. Thus we provide a semiuniform solution of the SAT problem in a polynomial number of steps by using mobile ambients with a weak form of replication which work according to a parallel semantics.

As far as we know, this is a first attempt to use mobile ambients with parallelism (as they were introduced initially) to create an algorithm that solves an NP-complete problem in a polynomial number of steps. In this way, we show how the mobile ambients can be coordinated to solve problems. There are several topics that could be investigated as further work, including finding other hard problems and complexity classes that can be solved using mobile ambients or related formalisms (process calculi).

Acknowledgements. The work of Bogdan Aman and Gabriel Ciobanu was supported by a grant of the Romanian National Authority for Scientific Research, CNCS-UEFISCDI, project number PN-II-ID-PCE-2011-3-0919.

\section{References}

1. Aman, B., Ciobanu, G.: Mobile Ambients with Timers and Types. In: Jones, C.B., Liu, Z., Woodcock, J. (eds.) ICTAC 2007. LNCS, vol. 4711, pp. 50-63. Springer, Heidelberg (2007)

2. Aman, B., Ciobanu, G.: Timed Mobile Ambients for Network Protocols. In: Suzuki, K., Higashino, T., Yasumoto, K., El-Fakih, K. (eds.) FORTE 2008. LNCS, vol. 5048, pp. 234-250. Springer, Heidelberg (2008)

3. Aman, B., Ciobanu, G.: Mobility in Process Calculi and Natural Computation. Springer (2011)

4. Bloom, B., Istrail, S., Meyer, A.R.: Bisimulation Can't Be Traced: Preliminary Report. In: 15th ACM Symposium on Principles of Programming Languages, pp. 229-239 (1988)

5. Boer, F., Gabbrielli, M., Meo, M.: A Timed Linda Language and its Denotational Semantics. Fundamenta Informaticae 63 (2004) 
6. Borodin, A.: On Relating Time and Space to Size and Depth. SIAM Journal of Computing 6, 733-744 (1977)

7. Bugliesi, M., Castagna, G., Crafa, S.: Access Control for Mobile Agents: the Calculus of Boxed Ambients. ACM Transactions on Programming and Systems 26, 57-124 (2004)

8. Busi, N.: On the Computational Power of the Mate/Bud/Drip Brane Calculus: Interleaving vs. Maximal Parallelism. In: Freund, R., Păun, G., Rozenberg, G., Salomaa, A. (eds.) WMC 2005. LNCS, vol. 3850, pp. 144-158. Springer, Heidelberg (2006)

9. Busi, N., Zavattaro, G.: On the Expressive Power of Movement and Restriction in Pure Mobile Ambients. Theoretical Computer Science 322, 477-515 (2004)

10. Cardelli, L.: Brane Calculi - Interactions of Biological Membranes. In: Danos, V., Schachter, V. (eds.) CMSB 2004. LNCS (LNBI), vol. 3082, pp. 257-278. Springer, Heidelberg (2005)

11. Cardelli, L., Gordon, A.: Mobile Ambients. Theoretical Computer Science 240, 177-213 (2000)

12. Charatonik, W., Gordon, A.D., Talbot, J.-M.: Finite-Control Mobile Ambients. In: Le Métayer, D. (ed.) ESOP 2002. LNCS, vol. 2305, pp. 295-313. Springer, Heidelberg (2002)

13. Ciobanu, G., Zakharov, V.A.: Encoding Mobile Ambients into the $\pi$-Calculus. In: Virbitskaite, I., Voronkov, A. (eds.) PSI 2006. LNCS, vol. 4378, pp. 148-165. Springer, Heidelberg (2007)

14. Fournet, C., Lévy, J.-J., Schmitt, A.: An Asynchronous, Distributed Implementation of Mobile Ambients. In: Watanabe, O., Hagiya, M., Ito, T., van Leeuwen, J., Mosses, P.D. (eds.) TCS 2000. LNCS, vol. 1872, pp. 348-364. Springer, Heidelberg (2000)

15. Garey, M., Johnson, D.: Computers and Intractability. A Guide to the Theory of NP-Completeness. Freeman (1979)

16. Teller, D., Zimmer, P., Hirschkoff, D.: Using Ambients to Control Resources. In: Brim, L., Jančar, P., Křetínský, M., Kučera, A. (eds.) CONCUR 2002. LNCS, vol. 2421, pp. 288-303. Springer, Heidelberg (2002)

17. Levi, F., Sangiorgi, D.: Mobile Safe Ambients. ACM Transactions on Programming and Systems 25, 1-69 (2003)

18. Maffeis, S., Phillips, I.: On the Computational Strength of Pure Ambient Calculi. Theoretical Computer Science 330, 501-551 (2005)

19. Milner, R.: Communicating and Mobile Systems: the $\pi$-Calculus. Cambridge University Press (1999)

20. Milner, R.: The Space and Motion of Communicating Agents. Cambridge University Press (2009)

21. Moller, F.: Axioms for Concurrency. PhD Thesis, Department of Computer Science, University of Edinburgh (1989)

22. Papadimitriou, C.H.: Computational Complexity. Addison-Wesley (1995)

23. Regev, A., Panina, E.M., Silverman, W., Cardelli, L., Shapiro, E.: BioAmbients: An Abstraction for Biological Compartments. Theoretical Computer Science 325, 141-167 (2004) 